\title{
Incidence and Economic Burden of
} Adverse Drug Reactions among Elderly Patients in Ontario Emergency Departments

\section{A Retrospective Study}

\author{
Chen Wu, ${ }^{1}$ Chaim M. Bell ${ }^{1,2,3,4,5}$ and Walter P. Wodchis ${ }^{1,4,6}$
}

1 Institute of Health Policy, Management and Evaluation, University of Toronto, Toronto, ON, Canada

2 Department of Medicine, University of Toronto, Toronto, ON, Canada

3 Keenan Research Centre in the Li Ka Shing Knowledge Institute, Toronto, ON, Canada

4 Institute for Clinical Evaluative Sciences, Toronto, ON, Canada

5 St Michael's Hospital, Toronto, ON, Canada

6 Toronto Rehabilitation Institute, Toronto, ON, Canada

\section{Abstract}

Background: The rapid rise in the availability and use of pharmaceutical agents, and particularly polypharmacy, directly increases the risk for patients to experience adverse drug reactions (ADRs). There are few studies on the overall incidence and costs of ADRs.

Objective: The aim of this study was to estimate the incidence and costs of emergency department (ED) visits related to ADRs for patients greater than 65 years of age using administrative data, and to describe risk factors for experiencing severe ADRs.

Methods: We employed a retrospective cohort design based on populationbased healthcare administrative clinical databases. Identification of ADRrelated ED visits from the administrative database was based on International Classification of Diseases, 10th Revision-Canadian Enhancement (ICD-10-CA) codes for each ED visit. The incidence and costs of ADR-related ED visits and subsequent hospital admissions were estimated for all adults aged 66 years and above for the period April 2003-March 2008. Costs were standardized and reported in 2008 Canadian dollars. Logistic regression was used to detect risk factors for severe ADRs.

Results: Approximately $0.75 \%$ of total annual ED visits among adults aged 66 years and above were found to be ADR-related, and among these patients $21.6 \%$ were hospitalized. In 2007, the cost of ADR-related visits was $\$ 333$ per ED visit and $\$ 7528$ per hospitalization for a total annual cost of $\$ 13.6$ million in Ontario, or an estimated $\$ 35.7$ million in Canada. Severe ADRs were associated with sex, age, comorbid disease burden, multiple drugs, multiple pharmacies, newly prescribed drugs, recent ED visit, recent hospitalization and long-term care (LTC) residence. 
Conclusions: ADRs are an important public health issue that threaten the safety of drug therapy and results in significant economic burden to the healthcare system. ED visits related to ADRs may be underestimated in retrospective studies using administrative data compared with prospective studies. Further research is needed to better understand the risk of experiencing severe ADRs among LTC residents.

\section{Background}

Several thousands of prescription medications are currently available. These drugs may cause adverse drug reactions (ADRs) defined by the WHO as "a response to a drug that is noxious and unintended and occurs at doses normally used in humans for the prophylaxis, diagnosis or therapy of disease, or for modification of physiological function". [1] ADRs can worsen a patient's medical problems, place patients in life-threatening situations and extend patients' length of stay in hospital (thus leading to increased healthcare costs). A landmark study by Lazarou and colleagues ${ }^{[2]}$ found ADRs to be among the top six leading causes of death in the US, with serious ADRs accounting for $6.7 \%$ of hospitalized admissions.

There are few observational studies of the population incidence and costs of ADRs. While some pharmacosurveillance studies include multicentre ${ }^{[3]}$ or national hospital data, ${ }^{[4]}$ most studies on ADRs have been conducted within a single hospital $^{[5]}$ or even a single medical department, ${ }^{[6]}$ making it difficult to confidently extrapolate results to a general population. ${ }^{[7]}$ As well, ADRs occur more commonly in community-dwelling patients ${ }^{[3]}$ where data is not well documented and more difficult to obtain. Finally, outpatients presenting to the emergency department (ED) for an ADR are typically not admitted, ${ }^{[8]}$ and have not been included in most previous studies. However, the loss of information from discharged ED patients may result in the loss of important epidemiological information on ADR incidence. We aimed to obtain population surveillance data for ADRs and an understanding of its economic burden. Specifically, we sought the rate of ADRs that can be observed in EDs and the proportion of severe ADRs that result in hospitalization or death. We also quantified the direct hospital care costs attributable to ADRs from the perspective of the single-payer government insurance plan. This population-based retrospective study examined the incidence and overall costs of ADRs among patients aged 65 years or older presenting to hospitals in Ontario, Canada.

\section{Methods}

\section{Data Sources}

Five linked, cross-sectional and provincially representative medical administrative databases were analysed, including the (i) Canadian Institute of Health Information (CIHI) National Ambulatory Care Reporting System (NACRS); (ii) CIHI Discharge Abstract Database (DAD); (iii) Ontario Drug Benefit Plan (ODB); (iv) Ontario Health Insurance Plan (OHIP); and (v) Ontario Registered Persons Database (RPDB).

NACRS includes data for all Ontario hospitalbased ambulatory care, including day surgery, outpatient clinics and EDs. The database contains demographic, clinical and service-specific data. The DAD includes patient-level data for all acute hospitalizations in Ontario. Up to 25 diagnosis codes using International Classification of Diseases, 10th Revision-Canadian Enhancement (ICD-10CA) are reported in this database, including one most responsible diagnosis code and other secondary diagnoses. ODB is a publicly funded comprehensive drug coverage plan for all Ontario residents over the age of 65 years. ODB provides the following information: drugs dispensed, dosage, days supplied, prescriber information and if the patient was resident in a long-term care (LTC) 
home at the time a prescription was issued. OHIP is a government-run health plan for the residents of Ontario that pays for all medically necessary physician, hospital and laboratory services. The OHIP dataset is based on physician billing records and contains the following information: physician identifiers, patient identifiers and fee codes for the medical service provided, diagnosis codes and date of service.

RPDB contains information on the vital status of all Ontario residents covered under OHIP. Demographic information in this study, including patient age, sex and income level were obtained from RPDB.

The above databases were linked through common encrypted health card numbers. The date of the ED visit was used as the index date. The administrative datasets used in this study have been used extensively for research about prescription medications. ${ }^{[9-11]}$

\section{Identification of Adverse Drug Reaction}

(ADR)-Related Emergency Department Visits

The study cohort included all Ontario patients greater than 65 years of age who presented to the ED with a condition or disorder related to ADRs between 1 April 2003 and 31 March 2007. NonOntario patients and patients without a valid Ontario health card number were excluded.

We based our identification of ADR-related ED visits on codes used in previous research from the UK. ${ }^{[4]}$ An ED visit record qualified as ADRrelated in one of two ways: (i) there was an ICD10-CA diagnosis code for ADR (Appendix table SI, Supplemental Digital Content [SDC], http://links. adisonline.com/DSZ/A73) listed in the 'Main Problem' or 'Other Problems' fields; or (ii) there was an ICD-10-CA code of external cause for ADR (Appendix table SII, online SDC) listed as 'Other Problems'. Any one indication was sufficient to identify an ADR visit.

\section{Analyses of Incidence of ADRs}

The initial ADR-related ED visits identified by the above method were defined as $\mathrm{ADR}_{\mathrm{ED}}$. For patients presenting to the ED with an ADR-related problem, after receiving diagnosis and manage- ment, three possible outcomes were identified: (i) hospitalization - the patient was admitted to hospital; (ii) death - the patient died during the ED visit; and (iii) discharge - the patient was discharged from the ED. These outcomes were defined as $\mathrm{ADR}_{\mathrm{ED}-\mathrm{ad}}, \mathrm{ADR}_{\mathrm{ED}-\text { died }}$ and $\mathrm{ADR}_{\mathrm{ED}-\text { discharged, }}$ respectively.

The incidence of $\mathrm{ADR}_{\mathrm{ED}}$ was calculated for each fiscal year (1 April-31 March of the following year) from 2003 to 2007. Incidence was expressed as the number of $\mathrm{ADR}_{\mathrm{ED}}$ divided by the total number of ED visits for patients greater than 65 years of age for each fiscal year. Within the cohort of $\mathrm{ADR}_{\mathrm{ED}}$, the incidence of $\mathrm{ADR}_{\mathrm{ED}-\mathrm{ad}}$, $\mathrm{ADR}_{\mathrm{ED} \text {-died }}$ and $\mathrm{ADR}_{\mathrm{ED} \text {-discharged were calcu- }}$ lated using the number of $\mathrm{ADR}_{\mathrm{ED}}$ in the corresponding fiscal year as the denominator. Trends over time were examined by comparing the incidence in each fiscal year.

\section{Analyses of Costs of ADRs}

All costs in EDs and inpatient medical care are paid by provincial single-payer insurance programmes in Canada. Individual inpatients may pay extra for private accommodation or amenities (telephone, television) but not medical care. The economic perspective for this study was therefore the Ontario single-payer government insurance programme. The economic burden of ADR-related hospital visits to the healthcare system was estimated by calculating direct medical care costs in two stages. Stage one included costs among patients with $\mathrm{ADR}_{\mathrm{ED}}$ receiving healthcare in the ED; stage two included hospitalrelated costs among patients admitted to hospital $\left(\mathrm{ADR}_{\mathrm{ED}-\mathrm{ad}}\right)$.

Costs for each ED visit and related hospital admissions were estimated by multiplying the patient's assigned Resource Intensity Weight (RIW) by the average provincial cost per weight (unit price) for the corresponding fiscal year. RIW is a measure of a patient's relative resource consumption compared with an average typical inpatient cost during a particular hospital separation. RIW is calculated annually from DAD, NACRS and Canadian case-cost data. ${ }^{[12]}$ RIW for the year 2008 was used for all ED visits and 
hospital admissions to make the data comparable. The total cost was the sum of the cost of all corresponding ED visits and hospital admissions. Unit prices were updated to the last observed period of data based on the Consumer Price Index for healthcare in Ontario ${ }^{[13]}$ and are expressed in 2008 Canadian dollars. A small number of patients presenting to the ED were admitted to hospital prior to the study end date of 31 March 2008 but discharged after the study end date. Because the database was censored at the study end date, costs could not be estimated for these patients but these patients were still included in other analyses.

\section{Risk Factors for Severe ADRs}

Patients who presented to the ED with an ADR-related problem and were then admitted to or died in the ED represent severe ADRs. These two categories of patients, i.e. $A_{D D} R_{E D-a d}$ and $\mathrm{ADR}_{\mathrm{ED}-\text { died, }}$ were combined and coded as $\mathrm{ADR}_{\text {severe. }}$ Patients who presented to the ED with an ADR and were subsequently discharged,

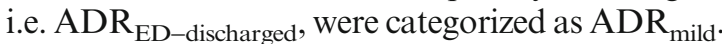

Multiple logistic regression analyses were applied to evaluate risk factors for experiencing severe ADRs for April 2007-March 2008. The regression model included measures of patient demographics, disease burden and care indicators: age, sex, income quintile, Charlson Comorbidity Index,${ }^{[14,15]}$ number of distinct prescription drugs/prescribers/pharmacies used in a 1-year period prior to the patient's visit to the ED, newly filled prescriptions, recent hospitalization, recent ED visit, whether the patients had a family physician and whether the patient resided in an LTC. Income quintiles (with 1 being the lowest) were calculated using neighbourhood income per person equivalent, which is the average household income adjusted by the household size based on 1996 Canadian census data at the level of enumeration area. ${ }^{[16]}$ The Charlson Comorbidity Index for each patient was calculated from a 5-year look-back for the 17 Charlson Comorbidity Diagnoses. The history of drug therapy was examined for each patient based upon a distinct prescription drug count from the 1-year period prior to the ED visit. The number of distinct prescribers and pharmacies were also calculated in this manner. Newly filled prescriptions included changes in brands for drugs with the same generic name, changes in doses for the same drug, as well as commencing new pharmaceutical agents within 30 days prior to the ED visit. Recent hospitalization/ED visit were identified if they occurred within 30 days prior to the current ED presentation.

\section{Statistical Analyses}

All statistical analyses were conducted using SAS for UNIX, version 9.1 (SAS Institute, Cary, NC, USA). Two-tailed $\mathrm{p}<0.05$ was considered statistically significant. Continuous variables were summarized using mean \pm SD. Categorical variables were presented as observed number and proportion. Continuous, ordinal and categorical variables were compared using the Student's t-test, the Wilcoxon rank test and the Chi-squared test, respectively.

A logistic regression model was first developed to evaluate odds ratios (ORs) and their 95\% confidence intervals (CIs) to predict severe ADRs based on all potential risk factors. Starting with the full model, including all measured independent variables, the backward selection method was conducted manually. Then, the final model was developed by eliminating all potential predictors that had no statistically significant regression coefficients $(p>0.10)$. Collinearity diagnostics first examined correlations among all independent variables, and then multiple linear regression with all independent variables in the final model was conducted to generate a variance inflation factor (VIF). VIF greater than 10 was used to assess collinearity among independent variables. ${ }^{[17]}$ Model diagnostics were conducted to test the goodness of fit, adequacy and accuracy of the final model. ORs with $95 \%$ CIs and the corresponding p-value were reported for all independent variables.

\section{Results}

\section{Analyses of Incidence of ADRs}

From 2003 through to 2007, the total ED visits in Ontario for patients aged 65 years and older 
increased from 826761 in 2003 to 966232 in 2007 similarly, the ADR-related ED visits increased from 6040 in 2003 to 7222 in 2007. (Each year includes data from April through to March in the subsequent year although we refer only to the first year for readability.) Among the ADR-related ED visits in 2007, 1250 reported the ADR as the main problem. ADR as an external-cause for a non-ADR main problem accounted for the remaining 5972 ED visits. For comparison, the population of Ontario reported in the 2006 census was 12.160 million people with $13.6 \%$ or approximately 1.65 million individuals aged 65 years and older. The total incidence of ADR-related ED visits ranged from $0.73 \%$ to $0.76 \%$, the incidence of ADR with diagnosis codes was $0.13-0.15 \%$, and the incidence of ADR with external cause codes was $0.67-0.70 \%$. Between 2003 and 2007, total ED visits for all causes increased by $16.87 \%$, while the total ADR-related ED visits increased by $19.57 \%$ (largely due to an increase in external cause codes), which indicates a slight increase in the proportion of ADR-related ED visits (table I).

The most common diagnoses (after 'unspecified adverse drug effect') in the ED for patients with ADRs (including both main reasons and external causes), was mental disorders due to multiple psychoactive drugs, mental disorders due to opioids and mental disorders due to sedatives or hypnotics, for all the patients with $\mathrm{ADR}_{\mathrm{ED}}$ and across patients experiencing severe ADRs and mild ADRs as well. In total, these three diagnoses accounted for $25 \%$ of severe ADRs and $33 \%$ of mild ADRs in 2007.

After being diagnosed and/or managed in the ED with an ADR-related condition, most patients were discharged. However at least one in five patients was admitted to hospital due to the severe ADRs, and a small portion of patients died in the ED. The proportion of patients admitted $(21 \%)$, discharged $(78 \%)$ or died $(0.07 \%)$ among all patients with $\mathrm{ADR}_{\mathrm{ED}}$ was approximately stable from 2003 through to 2007 (table II).

The most common principal diagnoses among inpatient admissions was agranulocytosis $(9.44 \%)$, followed by heart failure (3.64\%), abnormalities of heart beat $(3.24 \%)$, other coagulation defects $(3.17 \%)$ and poisoning by agents primarily affecting the cardiovascular system (3.03\%), which were directly or indirectly associated with heart diseases.

\section{Analyses of Costs of ADRs}

The average cost for each individual patient was stable from 2003 through to 2007. However due to the increasing volume of patients, the total cost in constant 2008 dollars for the cohort of patients with $\mathrm{ADR}_{\mathrm{ED}}$ increased $23 \%$ for those with ED visits only and $17 \%$ for those admitted to hospital (table III).

In 2007, 7222 patients with $\mathrm{ADR}_{\mathrm{ED}}$ cost the system $\$ 2408325$ for healthcare in the ED. While 5660 patients with mild ADRs cost \$235 per person for an ED visit, the average cost for those with severe ADRs was \$691. In addition, after being admitted to hospital, patients with severe ADRs cost an average $\$ 7529$ per person during their hospital stay. The total measured costs of ADR-related ED visits and subsequent hospitalizations amounted to \$13.6 million in 2007.

\section{Risk Factors for Severe ADRs}

There were 7222 ED visits for patients greater than 65 years of age identified with ADRs in

Table I. Total number of emergency department visits for which there was a diagnosis or external cause for adverse drug reactions 2003-7

\begin{tabular}{|c|c|c|c|c|c|c|}
\hline & $\begin{array}{l}2003 \\
{[n(\%)]}\end{array}$ & $\begin{array}{l}2004 \\
{[n(\%)]}\end{array}$ & $\begin{array}{l}2005 \\
{[n(\%)]}\end{array}$ & $\begin{array}{l}2006 \\
{[\mathrm{n}(\%)]}\end{array}$ & $\begin{array}{l}2007 \\
{[\mathrm{n}(\%)]}\end{array}$ & $\begin{array}{l}2003-7 \\
\text { (\% change) }\end{array}$ \\
\hline Total no. of ED visits & $826761(100)$ & $891027(100)$ & $910173(100)$ & $947799(100)$ & $966232(100)$ & 16.87 \\
\hline No. with diagnosis codes & $1231(0.15)$ & $1289(0.14)$ & $1332(0.15)$ & $1343(0.14)$ & $1250(0.13)$ & 1.54 \\
\hline No. with external cause codes & $5523(0.67)$ & $6058(0.68)$ & $6343(0.70)$ & $6622(0.70)$ & $6724(0.70)$ & 21.75 \\
\hline Total ADR visits $\left(A D R_{E D}\right)$ & $6040(0.73)$ & $6596(0.74)$ & $6930(0.76)$ & $7160(0.76)$ & $7222(0.75)$ & 19.57 \\
\hline
\end{tabular}

$\mathbf{A D R}=$ adverse drug reaction; $\mathbf{A D R}_{\mathrm{ED}}=$ initial $\mathrm{ADR}$-related $\mathrm{ED}$ visits; $\mathbf{E D}=$ emergency department. 
Table II. Outcome of patients who had adverse drug reaction-related emergency department visits

\begin{tabular}{lccccc}
\hline & $2003[\mathrm{n}(\%)]$ & $2004[\mathrm{n}(\%)]$ & $2005[\mathrm{n}(\%)]$ & $2006[\mathrm{n}(\%)]$ & $2007[\mathrm{n}(\%)]$ \\
\hline Total ADR visits & $6040(100)$ & $6596(100)$ & $6930(100)$ & $7160(100)$ & $7222(100)$ \\
Hospitalized & $1293(21.41)$ & $1358(20.58)$ & $1394(20.12)$ & $1497(20.91)$ & $1557(21.56)$ \\
Died in the ED & $5(0.08)$ & $<5(0.0)$ & $<5(0.0)$ & $5(0.07)$ & $5(0.07)$ \\
Discharged & $4742(78.51)$ & $5235(79.37)$ & $5534(79.86)$ & $5658(79.02)$ & $5660(78.37)$ \\
\hline
\end{tabular}

ADR = adverse drug reaction; ED=emergency department.

2007. Of these visits, 5660 (78.37\%) were judged as mild, while $1562(21.63 \%)$ were identified as having severe ADRs. Female patients represented $61 \%$ of ADR-related ED visits. The majority of patients had their own family physicians (95\%), while only $4 \%$ were LTC residents at the time of presenting to the ED. The mean age of the overall population was 77 years. The mean Charlson Cormobidity Index was 3.6. More than half of the patients $(60.0 \%)$ took over 11 distinct drugs in 1 year before they presented to the ED. In the same period, $85 \%$ of the patients had two or more distinct physician prescribers and $50 \%$ filled their prescriptions in different pharmacies.
Overall, 952 (13.2\%) had recent hospital admissions and 2560 (35.5\%) had recent ED visits. In the 30 days prior to their ED visits, a mean $( \pm \mathrm{SD})$ of $1.7 \pm 2.0$ new drugs were added to patients' drug therapy (table IV).

Patients with severe ADRs were older, sicker and more likely to take more than 11 drugs than those with mild ADRs. A higher proportion of patients with severe ADRs were male (44\%) compared with patients with mild ADRs ( $38 \%$ male); LTC residents were similarly more common among severe ADRs than among mild ADRs. However, there were no differences in income level and those having a family physician, but there were

Table III. Costs of adverse drug reaction-related emergency department visits and subsequent hospital admissions ${ }^{\mathrm{a}}$

\begin{tabular}{|c|c|c|c|c|c|c|c|}
\hline & & & 2003 & 2004 & 2005 & 2006 & 2007 \\
\hline \multirow{12}{*}{$\begin{array}{l}\text { Costs incurred } \\
\text { in the ED }\end{array}$} & \multirow[t]{4}{*}{$\mathrm{ADR}_{\mathrm{ED}}$} & Total cost $(\$)$ & 1965075 & 2117750 & 2221175 & 2368925 & 2408325 \\
\hline & & No. of visits & 6040 & 6596 & 6930 & 7160 & 7222 \\
\hline & & Average cost (\$) & 325 & 321 & 321 & 331 & 333 \\
\hline & & SD (\$) & 225 & 216 & 225 & 238 & 232 \\
\hline & \multirow[t]{4}{*}{$\mathrm{ADR}_{\text {severe }}$} & Total cost (\$) & 861875 & 906200 & 930825 & 1034250 & 1078575 \\
\hline & & No. of visits & 1298 & 1361 & 1396 & 1502 & 1562 \\
\hline & & Average cost (\$) & 664 & 666 & 667 & 689 & 691 \\
\hline & & $\mathrm{SD}(\$)$ & 224 & 175 & 227 & 238 & 196 \\
\hline & \multirow[t]{4}{*}{$A D R_{\text {mild }}$} & Total cost $(\$)$ & 1103200 & 1211550 & 1290350 & 1334675 & 1329750 \\
\hline & & No. of visits & 4742 & 5235 & 5534 & 5658 & 5660 \\
\hline & & Average cost (\$) & 233 & 231 & 233 & 236 & 235 \\
\hline & & $\mathrm{SD}(\$)$ & 104 & 109 & 113 & 117 & 117 \\
\hline \multirow{4}{*}{$\begin{array}{l}\text { Costs incurred } \\
\text { in hospital }\end{array}$} & \multirow[t]{4}{*}{$\mathrm{ADR}_{\mathrm{ED}-\mathrm{ad}}$} & Total cost $(\$)$ & 9544650 & 10027300 & 9840150 & 11426000 & 11164975 \\
\hline & & No. of admissions & 1289 & 1357 & 1380 & 1471 & 1483 \\
\hline & & Average cost (\$) & 7405 & 7389 & 7131 & 7768 & 7529 \\
\hline & & $\mathrm{SD}(\$)$ & 9571 & 12187 & 9342 & 11937 & 11900 \\
\hline
\end{tabular}

a All costs in 2008 Canadian dollars.

$\mathbf{A D R}=$ adverse drug reaction; $\mathbf{A D R}_{\mathrm{ED}-\mathbf{a d}}=\mathrm{ADR}$ diagnosis in $\mathrm{ED}$ and then admitted to acute care; $\mathbf{A D R} \mathbf{R}_{\mathrm{ED}}=$ overall $\mathrm{ADR}$-related $\mathrm{ED}$ visits; $\mathbf{A D R}_{\text {mild }}=$ admitted to ED with $\mathrm{ADR}$ and discharged home; $\mathbf{A D R}_{\text {severe }}=$ admitted to $E D$ with $\mathrm{ADR}$ and then either admitted or died; ED = emergency department; $\mathbf{S D}=$ standard deviation. 
Table IV. Characteristics of patients having adverse drug reactionrelated emergency department visits in 2007

\begin{tabular}{|c|c|}
\hline Variable & $\begin{array}{l}\text { Prevalence or mean }( \pm S D) \\
\text { among patients with } A R_{E D} \\
(n=722)^{a}\end{array}$ \\
\hline Having severe ADRs & $1562(21.6)$ \\
\hline Female & $4382(60.7)$ \\
\hline Age (years) & $77.0 \pm 7.1$ \\
\hline \multicolumn{2}{|l|}{ Charlson Comorbidity Index } \\
\hline 0 & $923(12.8)$ \\
\hline 1 & $1219(16.9)$ \\
\hline 2 & $1263(17.5)$ \\
\hline $3+$ & $3814(52.8)$ \\
\hline \multicolumn{2}{|l|}{$\begin{array}{l}\text { Number of drugs used in a } \\
1 \text {-year period prior to index }\end{array}$} \\
\hline $0-5$ & 829 (11.5) \\
\hline $6-10$ & $2062(28.6)$ \\
\hline $11+$ & $4331(60.0)$ \\
\hline \multicolumn{2}{|l|}{$\begin{array}{l}\text { Number of prescribers in a } \\
1 \text {-year period prior to index }\end{array}$} \\
\hline $0-1$ & $1117(15.5)$ \\
\hline $2+$ & $6105(84.5)$ \\
\hline \multicolumn{2}{|l|}{$\begin{array}{l}\text { Number of pharmacies used in a } \\
1 \text {-year period prior to index }\end{array}$} \\
\hline $0-1$ & $3644(50.5)$ \\
\hline $2+$ & $3578(49.5)$ \\
\hline \multicolumn{2}{|l|}{$\begin{array}{l}\text { Number of new drugs within } \\
30 \text { days prior to index }\end{array}$} \\
\hline 0 & $2159(29.9)$ \\
\hline 1 & $2007(27.8)$ \\
\hline 2 & $1243(17.2)$ \\
\hline 3 & $751(10.4)$ \\
\hline 4 & $443(6.1)$ \\
\hline $5+$ & $619(8.6)$ \\
\hline Recent hospital admission (Yes) & $952(13.2)$ \\
\hline Recent ED visit (Yes) & $2560(35.5)$ \\
\hline Family physician (Yes) & $6858(95.0)$ \\
\hline Long-term care resident (Yes) & $290(4.0)$ \\
\hline
\end{tabular}

$\mathbf{A D R}(\mathbf{s})=$ adverse drug reaction(s); $\mathbf{A D R}_{\mathrm{ED}}=$ initial $\mathrm{ADR}$-related $\mathrm{ED}$ visits; ED = emergency department.

strong significant differences in other risk factors (table V).

Consistent with the results of univariate analysis, multiple logistic regression demonstrated that females were less likely to have severe ADRs than males (table VI: adjusted OR 0.81; 95\% CI
$0.72,0.92)$. Age was an independent risk factor for severe ADRs and the odds of experiencing severe ADRs increased by $3 \%$ per 1 -year increase in age. Severe ADRs occurred more frequently in patients who had many coexisting conditions. Compared with those with no Charlson comorbidities, a Charlson Index score of 3 or more was associated with an adjusted OR for severe ADR of $1.86(95 \%$ CI $1.48,2.33)$. The number of distinct prescription drugs and the number of pharmacies used in the last year before the ED visit were both risk factors for severe ADRs. Compared with patients who had $0-5$ drugs, patients taking 6-10 drugs and more than 11 drugs experienced a risk of severe ADRs that increased by $48 \%$ and $93 \%$, respectively. Patients who filled prescriptions in multiple pharmacies had a higher risk for severe ADRs (adjusted OR 1.13; 95\% CI $1.00,1.27)$. Patients were less likely to have severe ADRs if they had one or two new drugs in their drug therapy. However, when more new drugs were added, the protective effect decreased and was eliminated when there were more than five new drugs. The odds of having severe ADRs increased by $47 \%$ if the patient had a prior hospitalization within 30 days prior to the index ED visit. In contrast, the odds of having severe ADRs decreased by $27 \%$ if the patient had an ED visit without hospitalization within 30 days prior to the index ED visit. LTC residents were 2.08 times more likely to experience severe ADRs than community residents. No multi-collinearity was detected in the final variable set. The Hosmer and Lemeshow goodness-of-fit test's p-value was larger than 0.05 , indicating that the model predicted the data well, and the c-statistic was 0.667. ${ }^{[18]}$

\section{Discussion}

This is the first population-based research to study ADR-related ED visits and subsequent hospital admissions using administrative data in Canada. This study found that the incidence of ADR-related ED visits with patients greater than 65 years of age was stable at approximately $0.75 \%$ of all ED visits from 2003 through to 2007, and the average cost for an individual patient with ADR-related problems was similarly stable. As a 
result of the increasing number of ADR-related acute admissions, total costs increased significantly in constant dollars. In 2007, the total measured costs of ADR-related ED visits and subsequent hospitalizations in Ontario amounted to $\$ 13.6$ million. If ED presentation for ADR and severity among adults over 65 years is similar across Canada, the national annual estimated cost is $\$ 35.7$ million or $\$ 7$ per adult over age 65 years. Our study of 7222 elderly patients found that severe ADRs were associated with sex, age, comorbidity, multiple drugs, multiple pharmacies,

Table V. Characteristics of patients having adverse drug reaction-related emergency department visits in 2007 according to the severity of the adverse drug reactions ${ }^{a}$

\begin{tabular}{|c|c|c|c|}
\hline Variable & $\begin{array}{l}\text { Patients with severe } \\
\text { ADRs }(n=1562)[n(\%)]\end{array}$ & $\begin{array}{l}\text { Patients with mild } \\
\text { ADRs }(n=5660)[n(\%)]\end{array}$ & $\begin{array}{l}\text { Significance } \\
\text { (p-value) }\end{array}$ \\
\hline Female & $878(56.2)$ & $3504(61.9)$ & $<0.0001$ \\
\hline Age (years) & $78.4 \pm 7.5$ & $76.6 \pm 7.0$ & $<0.0001$ \\
\hline Income quintile & & & NS \\
\hline 1 & $375(24.0)$ & $1306(23.1)$ & \\
\hline 2 & 349 (22.3) & $1241(21.9)$ & \\
\hline 3 & $282(18.1)$ & $1156(20.4)$ & \\
\hline 4 & $293(18.6)$ & $993(17.5)$ & \\
\hline 5 & $251(16.1)$ & $929(16.4)$ & \\
\hline Charlson Comorbidity Index & & & $<0.0001$ \\
\hline 0 & $112(7.2)$ & $811(14.3)$ & \\
\hline 1 & $157(10.1)$ & $1062(18.8)$ & \\
\hline 2 & $234(15.0)$ & $1029(18.2)$ & \\
\hline $3+$ & $1059(67.8)$ & $2755(48.7)$ & \\
\hline Number of drugs used in a 1-year period prior to index & & & $<0.0001$ \\
\hline $0-5$ & $87(5.6)$ & $742(13.1)$ & \\
\hline $6-10$ & $351(22.5)$ & $1711(30.2)$ & \\
\hline $11+$ & $1124(72.0)$ & $3207(56.7)$ & \\
\hline Number of prescribers in a 1-year period prior to index & & & 0.0008 \\
\hline $0-1$ & $199(12.7)$ & $918(16.2)$ & \\
\hline $2+$ & $1363(87.3)$ & $4742(83.8)$ & \\
\hline Number of pharmacies used in a 1-year period prior to index & & & $<0.0001$ \\
\hline $0-1$ & $716(45.8)$ & $2928(51.7)$ & \\
\hline $2+$ & $846(54.2)$ & $2732(48.3)$ & \\
\hline Number of new drugs within 30 days prior to index & & & $<0.0001$ \\
\hline 0 & $477(30.5)$ & $1682(29.7)$ & \\
\hline 1 & $364(23.3)$ & $1643(29.3)$ & \\
\hline 2 & $229(14.7)$ & $1014(17.9)$ & \\
\hline 3 & $178(11.4)$ & $573(10.1)$ & \\
\hline 4 & $109(7.0)$ & $334(5.9)$ & \\
\hline $5+$ & $205(13.1)$ & $414(7.3)$ & \\
\hline Recent hospital admission (Yes) & $287(18.4)$ & $665(11.8)$ & $<0.0001$ \\
\hline Recent ED visit (Yes) & $512(32.8)$ & $2048(36.3)$ & 0.0127 \\
\hline Family physician (Yes) & $1499(96.0)$ & $5359(94.7)$ & NS \\
\hline Long-term care resident (Yes) & $131(8.4)$ & $159(2.8)$ & $<0.0001$ \\
\hline \multicolumn{4}{|c|}{ Continuous data presented as mean \pm SD; statistical significance test was done by two independent sample t-tests. } \\
\hline b Categorical data presented as observed number (proport & statistical significance te & done by Chi-squared tes & \\
\hline ADRs = adverse drug reactions; $\mathbf{E D}=$ emergency departmer & not significant. & & \\
\hline
\end{tabular}


Table VI. Odds ratio of risk factors for experiencing severe adverse drug reactions

\begin{tabular}{|c|c|c|c|c|}
\hline \multirow[t]{2}{*}{ Variable } & \multicolumn{2}{|c|}{ Full model $(n=7179)$} & \multicolumn{2}{|c|}{ Final model $(n=7179)$} \\
\hline & OR $(95 \% \mathrm{Cl})$ & $p$-Value & OR $(95 \% \mathrm{Cl})$ & $\mathrm{p}$-Value \\
\hline Female & $0.81(0.72,0.92)$ & 0.0007 & $0.81(0.72,0.92)$ & 0.0006 \\
\hline Age (years) & $1.03(1.02,1.04)$ & $<0.0001$ & $1.03(1.02,1.04)$ & $<0.0001$ \\
\hline \multicolumn{5}{|l|}{ Income quintile } \\
\hline 1 & Reference & & & \\
\hline 2 & $1.01(0.85,1.20)$ & 0.9284 & & \\
\hline 3 & $0.91(0.76,1.09)$ & 0.2902 & & \\
\hline 4 & $1.05(0.88,1.26)$ & 0.5808 & & \\
\hline 5 & $1.00(0.83,1.21)$ & 0.9972 & & \\
\hline \multicolumn{5}{|l|}{ Charlson Comorbidity Index } \\
\hline 0 & Reference & & Reference & \\
\hline 1 & $0.91(0.70,1.19)$ & 0.4935 & $0.91(0.70,1.19)$ & 0.4899 \\
\hline 2 & $1.24(0.96,1.60)$ & 0.0953 & $1.24(0.97,1.60)$ & 0.0921 \\
\hline $3+$ & $1.84(1.47,2.31)$ & $<0.0001$ & $1.86(1.48,2.33)$ & $<0.0001$ \\
\hline \multicolumn{5}{|c|}{ Number of drugs used in a 1-year period prior to index } \\
\hline $0-5$ & Reference & & Reference & \\
\hline $6-10$ & $1.46(1.11,1.90)$ & 0.0061 & $1.48(1.13,1.93)$ & 0.0038 \\
\hline $11+$ & $1.88(1.44,2.46)$ & $<0.0001$ & $1.93(1.49,2.51)$ & $<0.0001$ \\
\hline \multicolumn{5}{|c|}{ Number of prescribers used in a 1-year period prior to index } \\
\hline $0-1$ & Reference & & & \\
\hline $2+$ & $1.08(0.90,1.31)$ & 0.4007 & & \\
\hline \multicolumn{5}{|c|}{ Number of pharmacies used in a 1-year period prior to index } \\
\hline $0-1$ & Reference & & Reference & \\
\hline $2+$ & $1.11(0.99,1.26)$ & 0.08 & $1.13(1.00,1.27)$ & 0.0532 \\
\hline \multicolumn{5}{|c|}{ Number of new drugs within 30 days prior to index } \\
\hline 0 & Reference & & Reference & \\
\hline 1 & $0.75(0.64,0.88)$ & 0.0005 & $0.75(0.64,0.88)$ & 0.0005 \\
\hline 2 & $0.71(0.59,0.85)$ & 0.0003 & $0.71(0.59,0.86)$ & 0.0003 \\
\hline 3 & $0.94(0.76,1.16)$ & 0.5865 & $0.95(0.77,1.17)$ & 0.6381 \\
\hline 4 & $0.92(0.71,1.18)$ & 0.5003 & $0.92(0.72,1.19)$ & 0.5391 \\
\hline $5+$ & $1.16(0.93,1.46)$ & 0.1948 & $1.17(0.93,1.47)$ & 0.1822 \\
\hline Recent hospital admission (Yes) & $1.47(1.23,1.76)$ & $<0.0001$ & $1.47(1.23,1.76)$ & $<0.0001$ \\
\hline Recent ED visit (Yes) & $0.73(0.64,0.84)$ & $<0.0001$ & $0.73(0.64,0.84)$ & $<0.0001$ \\
\hline Family physician (Yes) & $1.30(0.95,1.79)$ & 0.6864 & & \\
\hline Long-term care resident (Yes) & $2.10(1.63,2.72)$ & $<0.0001$ & $2.08(1.62,2.67)$ & $<0.0001$ \\
\hline
\end{tabular}

newly prescribed drugs, recent ED visits, recent hospitalizations and LTC residents.

The strengths of our study include the longitudinal nature and its comprehensiveness. The incidence and costs of ADR-related ED visits were studied over a 5-year period. Our study included all ED visits and all acute hospitalizations in Ontario. Compared with studies conducted within a single medical unit or hospital, we provided estimates at the population level without the issue of lack of representativeness. Our results are also likely to be representative of care settings across Canada. Prospective analyses and chart reviews of ED visits from across the country have 
found consistent results in the incidence of ADRs and presentation to ED. ${ }^{[19,20]}$ An important weakness with the retrospective analysis of administrative databases is that we are not able to identify which specific drug was the cause for ED visits or the inpatient admissions.

The estimate of incidence of ADR-related ED visits in this study is nearly 2 -fold higher than that found in a UK-based retrospective study that reported that $0.46 \%$ of ED visits were related to ADRs. ${ }^{[21]}$ The fact that the study population in this study was exclusively seniors while the latter study included patients of all ages could be one reason for this difference. Seniors generally have more comorbidities and thus take more concurrent drugs.

Although not directly comparable, an incidence of $0.75 \%$ is substantially lower than those from single-hospital prospective studies, suggesting there is considerable underestimation of ADRs in administrative databases. A study from the US showed that of $253 \mathrm{ED}$ visits, $8.37 \%$ were for ADRs. ${ }^{[22]}$ An Indian study found $2.66 \%$ of the total 4764 patients presented to the ED because of ADRs. ${ }^{[23]}$ Another Indian study of patients greater than age 65 years showed $6.7 \%$ of the 578 ED visits were caused by ADRs. ${ }^{[2]}$ A recent Canadian study reported that $12.0 \%(122 / 1017)$ of the ED visits were judged as drug-related, while the most common reason for drug-related visits were ADRs (39.3\%). ${ }^{[25]}$

Several reasons may account for the underestimation. Firstly, with any study of retrospective design, there is a potential for underestimating the real incidence because routinely collected data could be incomplete, inaccurate or missing. Secondly, our study could not identify all ADRrelated ED visits due to the limitation of the ICD codes used. The external causes for ADR cover almost all drug classes that are administered in current clinical practice, but the diagnosis codes related to ADR cannot cover all potential illnesses that might be caused by ADRs. Among cases that were identified by codes of external cause, only $10 \%$ had records of diagnosis related to ADR. This reflects another limitation of our study that causality could not be established without examining detailed information on drug history and symptoms. Although no studies have examined the sensitivity and specificity of the diagnosis of ADR in DAD or NACRS, a validation study based on chart re-abstraction reported that most responsible diagnoses were fairly accurate across the DAD. ${ }^{[26]}$ Another validation study of the ODB reported an overall error rate of $0.7 \%$ with regards to the coding of drug identifiers, date dispensed, quantity dispensed and duration of dispensation. ${ }^{[27]}$ RPDB was reported to have good information on sex and birth dates. ${ }^{[28]}$ Thirdly, another issue relates to underdiagnosis by physicians and is related to the current practice pattern in the ED. ${ }^{[17]}$ While prospective studies always employ computerized monitoring programmes and trained professionals to detect ADRs, ED physicians are responsible for identifying ADRs. Since new drugs are entering the market at a rapid rate, it is impractical for any physician to be familiar with all the drugs and identify countless associated ADRs, especially when working in a stressed environment. Poor recognition could account for the low estimate of the incidence of ADR-related ED visits as noted in a German study that more than half of the preexisting ADRs were not recognized by either the admitting or the attending physician. ${ }^{[5]}$ Finally, we note that there is likely to be facility-level variability in identification and coding of ADRs in the CIHI databases used in this analysis. Although we do not believe such variability would be a confounding factor in the present analysis, future work could investigate such variability.

An important finding is the substantial cost for patients with severe ADRs. Patients with severe ADRs had ED costs almost three times greater than patients with mild ADRs, and after being admitted to hospital, these patients with severe ADRs cost an additional \$11 million per year. This result provides support to the rationale of this study in exploring risk factors for severe ADRs. Identifying and potentially reducing the risks of severe ADRs could result in significant cost reductions for hospital care. Costs reported here relate to hospitalizations that resulted from the onset of an ADR. Some costs for inpatient hospital stays may have been incurred in providing treatment for management of conditions not exclusively related to the ADR. At the same time, 
this study does not include incremental costs associated with ADRs among patients admitted to hospital for non-ADR reasons, as has been done in earlier work. ${ }^{[29]}$

While some studies have explored factors associated with the occurrence of all ADRs, this study also investigated risk factors for (severe) ADRs presenting in the ED that required hospitalization. Any drug could cause an ADR even when the correct drug is administered properly at the normal dose. In reality, some mild or even moderate ADRs, such as rash due to antibiotics or nausea and vomiting due to antineoplastic agents, may be reasonably tolerated as long as the drug's therapeutic benefit outweighs the known adverse effects. However, when severe ADRs happen, it raises the question of whether the harm of a drug outweighs the benefit. This study makes an important contribution by investigating risk factors for severe ADRs, including sex, age, comorbidity, multiple drugs, multiple pharmacies, newly prescribed drugs, recent ED visit, recent hospitalization and LTC residence.

Consistent with previous work, ${ }^{[30-33]}$ this study identified ADRs to be more common among females $(60.7 \%$ of the overall ADR-related ED visits) than males. However, females appear to have a lower risk for experiencing severe ADRs. This may indicate that females seek medical care earlier than males. Some studies demonstrated that when confined to the geriatric age group in which multiple co-morbidities and multiple medications are the norm, age itself is not an independent risk factor for ADR. ${ }^{[34,35]}$ However, our findings indicated that age itself is an independent risk factor for severe ADRs after adjustment for co-morbidities and multiple medications. This is supported by higher rates reported by Leendertse and colleagues ${ }^{[7]}$ where ADR rates among the elderly were $3.6 \%$ compared with $3.0 \%$ among all hospital admissions.

There is evidence that using multiple medications can predispose patients to drug-related problems such as ADRs, drug-drug interactions and non-compliance. ${ }^{[36-38]}$ In our study the average number of different drug prescriptions filled in the year prior to presenting to the ED was 12.9 (not necessarily all simultaneously), and multiple medications was identified as a risk factor for severe ADRs. The effect of multiple medications can also explain why large numbers of drug changes and the introduction of new drugs within 30 days prior to the ED visit were risk factors for severe ADRs, while small numbers of drug changes had a protective effect. Drug changes could be a result of a recent physician visit, which could also provide an opportunity for timely detection and intervention on potential medication problems.

Filling prescriptions at different pharmacies may be dangerous to patients. Newly added drugs may interact with older drugs kept in a patient's medication regimen and result in drug-related problems. Pharmacists are trained to identify and resolve potential drug-related problems but currently different pharmacies in Ontario do not share information on a patient's medication history. Other clinical interventions that could potentially reduce the incidence and burden of ADRs include using a preferred prescriber and medication reconciliation interventions that include patient education regarding their medications. ${ }^{[39-42]}$

LTC residents were found to be about 2-fold more likely to experience severe ADRs than people living at home. Residents of LTC homes are a particularly vulnerable population as they often have multiple morbidities and cognitive impairment. ${ }^{[43]}$ Although this study controlled for risk factors such as age, comorbidity and multiple drugs, cognitive impairment could be an unmeasured contributor. Cognitive impairment has been reported to be a risk factor for adverse drug events because patients may have difficulty in understanding instructions for medications and in managing multiple medications. ${ }^{[44]}$ Among LTC residents, cognitive impairment may limit their capacity to report symptoms and adverse reactions will not be detected early.

\section{Conclusions}

ADRs are an important public health issue that threaten the safety of drug therapy and results in significant economic burden to the healthcare system. With far lower rates than those found in 
prospective or trial-based results, our study suggests that ED visits related to ADRs are not well identified or coded in emergency settings. Since early identification of an ADR is the predominant factor in preventability, ${ }^{[5]}$ effective interventions aimed at improving ADR identification are warranted.

\section{Acknowledgements}

This research was supported through funding from the Health System Performance Research Network (of which Walter Wodchis is the primary investigator) and conducted at the Institute for Clinical Evaluative Sciences, supported in part by grants from the Ontario Ministry of Health and LongTerm Care. The opinions, results, and conclusions are those of the authors with no endorsement by the Ontario Ministry of Health and Long-Term Care. Ms Wu and Dr Bell have no conflicts of interest to declare that are directly relevant to the content of this study.

\section{References}

1. WHO. International drug monitoring: the role of the national centers. World Health Organ Tech Rep Ser 1972; 498: 1-25

2. Lazarou J, Pomeranz BH, Corey PN. Incidence of adverse drug reactions in hospitalized patients: a meta-analysis of prospective studies. JAMA 1998 Apr 15; 279 (15): 1200-5

3. Onder G, Pedone C, Landi F, et al. Adverse drug reactions as cause of hospital admissions: results from the Italian Group of Pharmacoepidemiology in the Elderly (GIFA). J Am Geriatr Soc 2002 Dec; 50 (12): 1962-8

4. Patel H, Bell D, Molokhia M, et al. Trends in hospital admissions for adverse drug reactions in England: analysis of national hospital episode statistics 1998-2005. BMC Clin Pharmacol 2007; 7: 9

5. Dormann H, Criegee-Rieck M, Neubert A, et al. Lack of awareness of community-acquired adverse drug reactions upon hospital admission : dimensions and consequences of a dilemma. Drug Saf 2003; 26 (5): 353-62

6. Moore N, Lecointre D, Noblet C, et al. Frequency and cost of serious adverse drug reactions in a department of general medicine. Br J Clin Pharmacol 1998 Mar; 45 (3): 301-8

7. Leendertse AJ, Visser D, Egberts ACG, et al. The relationship between study characteristics and the prevalence of medication-related hosptializations. Drug Saf 2010; 33 (3): 233-44

8. Zed PJ. Drug-related visits to the emergency department. J Pharm Pract 2005 October 1; 18 (5): 329-35

9. Lipscombe LL, Gomes T, Levesque LE, et al. Thiazolidinediones and cardiovascular outcomes in older patients with diabetes. JAMA 2007 Dec 12; 298 (22): 2634-43

10. Austin PC, Donovan L, Yun L, et al. Comparing clinical and administrative data for profiling hospitals on postdischarge medication use by patients with acute myocardial infarction. Am Heart J 2008 9; 156 (3): 595-605

11. Juurlink DN, Gomes T, Ko DT, et al. A population-based study of the drug interaction between proton pump inhibitors and clopidogrel. CMAJ 2009 Mar 31; 180 (7): 713-8
12. Canadian Institute for Health Information. $\mathrm{CMG}+$ tool kit: transitioning to the new $\mathrm{CMG}+$ grouping methodology (and associated health resource indicators) fiscal 20072008. Toronto (ON): Canadian Institute for Health Information, 2007

13. Consumer Price Index, health and personal care, by province (Ontario) [online]. Available from URL: http:/ www40.statcan.ca/101/cst01/econ161g-eng.htm [Accessed 2011 Jul 21]

14. Charlson ME, Pompei P, Ales KL, et al. A new method of classifying prognostic comorbidity in longitudinal studies: development and validation. J Chronic Dis 1987; 40 (5): 373-83

15. Deyo RA, Cherkin DC, Ciol MA. Adapting a clinical comorbidity index for use with ICD-9-CM administrative databases. J Clin Epidemiol 1992 Jun; 45 (6): 613-9

16. Statistics Canada. Census Tract (CT) profiles, 2006 census. Catalogue 92-597-XWE. Ottawa (ON) : Statistics Canada, 2007

17. Kutner M, Nachtsheim C, Neter N, Applied linear regression Models. 4th ed. New York: McGraw-Hill Irwin, 2004

18. Allison $\mathrm{P}$, editor. Logistic regression using the SAS system: theory and application. Cary (NC): SAS Institute, 2001

19. Forster AJ, Clark HD, Menard A, et al. Adverse events among medical patients after discharge from hospital. CMAJ 2004 Feb 3; 170 (3): 345-9

20. Zed PJ, Abu-laban AB, Balen RM, et al. Incidence, severity and preventability of medication-related visits to the emergency department: a prospective study. CMAJ 2008 June 3; 178 (12): 1563-9

21. Smith KM, McAdams JW, Frenia ML, et al. Drug-related problems in emergency department patients. Am J Health Syst Pharm 1997 Feb 1; 54 (3): 295-8

22. Tafreshi MJ, Melby MJ, Kaback KR, et al. Medicationrelated visits to the emergency department: a prospective study. Ann Pharmacother 1999 Dec; 33 (12): 1252-7

23. Malhotra S, Jain S, Pandhi P. Drug-related visits to the medical emergency department: a prospective study from India. Int J Clin Pharmacol Ther 2001 Jan; 39 (1): 12-8

24. Malhotra S, Karan RS, Pandhi P, et al. Drug related medical emergencies in the elderly: role of adverse drug reactions and non-compliance. Postgrad Med J 2001 Nov; 77 (913): 703-7

25. Zed PJ, Abu-Laban RB, Balen RM, et al. Incidence, severity and preventability of medication-related visits to the emergency department: a prospective study. CMAJ 2008 Jun 3; 178 (12): 1563-9

26. Juurlink D, Preyra C, Croxford R, et al. Canadian Institute for Health Information discharge abstract database: a validation study. Toronto (ON): Institute for Clinical Evaluative Sciences, 2006

27. Levy AR, O'Brien BJ, Sellors C, et al. Coding accuracy of administrative drug claims in the Ontario Drug Benefit database. Canadian J Clin Pharmacol 2003; 10 (2): 67-71

28. Institute for Clinical Evaluative Sciences. Improving health care data in Ontario. ICES investigative report. Toronto $(\mathrm{ON})$ : Institute for Clinical Evaluative Sciences, 2005

29. Gautier S, Bachelet H, Bordet R, et al. The cost of adverse drug reactions. Expert Opin Pharmacother 2003; 4 (3): 319-26

30. Fattinger K, Roos M, Vergeres P, et al. Epidemiology of drug exposure and adverse drug reactions in two swiss de- 
partments of internal medicine. Br J Clin Pharmacol 2000 Feb; 49 (2): 158-67

31. Pirmohamed M, James S, Meakin S, et al. Adverse drug reactions as cause of admission to hospital: prospective analysis of 18820 patients. BMJ 2004 Jul 3; 329 (7456): 15-9

32. van der Hooft CS, Sturkenboom MC, van Grootheest K, et al. Adverse drug reaction-related hospitalisations: a nationwide study in the Netherlands. Drug Saf 2006; 29 (2): 161-8

33. Puche Canas E, Luna Del Castillo JD. Adverse drug reactions in patients visiting a general hospital: a meta-analysis of results. An Med Interna 2007 Dec; 24 (12): 574-8

34. Mannesse CK, Derkx FH, de Ridder MA, et al. Adverse drug reactions in elderly patients as contributing factor for hospital admission: cross sectional study. BMJ 1997 Oct 25; 315 (7115): 1057-8

35. Carbonin P, Pahor M, Bernabei R, et al. Is age an independent risk factor of adverse drug reactions in hospitalized medical patients? J Am Geriatr Soc 1991 Nov; 39 (11): 1093-9

36. Bergman U, Wiholm BE. Drug-related problems causing admission to a medical clinic. Eur J Clin Pharmacol 1981; 20 (3): 193-200

37. Atkin PA, Shenfield GM. Medication-related adverse reactions and the elderly: a literature review. Adverse Drug React Toxicol Rev 1995; 14 (3): 175-91

38. Nguyen JK, Fouts MM, Kotabe SE, et al. Polypharmacy as a risk factor for adverse drug reactions in geriatric nursing home residents. Am J Geriatr Pharmacother 2006 Mar; 4 (1): $36-41$
39. Schnipper JL, Hamann C, Ndumele CD, et al. Effect of an electronic medication reconciliation application and process redesign on potential adverse drug events: a clusterrandomized trial. Arch Intern Med 2009; 169: 771-80

40. Schnipper JL, Kirwin JL, Cotugno MC, et al. Role of pharmacist counselling in preventing adverse drug events after hospitalization. Arch Intern Med 2006; 166: 565-71

41. Rogers G, Alper E, Brunelle D, et al. Reconciling medications at admission: safe practice recommendations and implementation strategies. Jt Comm J Qual Patient Saf. 2006; 32: 37-50

42. Waterman AD, Gallagher TH, Garbutt J, et al. Hospitalized patients' attitudes about and participation in error prevention. J Gen Intern Med 2006; 21: 367-70

43. Ontario Ministry of Health and Long-Term Care. 2006 levels of care classification: provincial summary data. Toronto (ON): Ontario Ministry of Health and Long-Term Care, 2007

44. Gray SL, Mahoney JE, Blough DK. Adverse drug events in elderly patients receiving home health services following hospital discharge. Ann Pharmacother 1999 Nov; 33 (11): 1147-53

Correspondence: Dr Walter P. Wodchis, Associate Professor, Institute of Health Policy, Management and Evaluation, Faculty of Medicine, University of Toronto, Health Sciences Building, 4th floor, 155 College Street, Suite 425, Toronto, ON M5T 3M6, Canada.

E-mail: walter.wodchis@utoronto.ca 